\title{
Agent Selection Strategies in Wireless Networks with Multihomed Mobile IP
}

\author{
Christer Åhlund ${ }^{1}$, Robert Brännström ${ }^{1}$, Arkady Zaslavsky ${ }^{2}$ \\ ${ }^{1}$ Luleå University of Technology, Department of Computer Science, SE-971 87 Luleå, Sweden \\ \{christer.ahlund, robert.brannstrom\}@ltu.se \\ ${ }^{2}$ School of Computer Science \& Software Engineering, Monash University, \\ 900 Dandenong Road, Caulfield East, \\ Vic 3145, Melbourne, Australia \\ a.zaslavsky@monash.edu.au
}

\begin{abstract}
Mobile IP is a proposed standard for mobility management in IP networks. With today's emerging possibilities within wireless broadband communication, mobility within networks will increase. New applications and protocols will be created and Mobile IP is important to this development, since Mobile IP support is needed to allow mobile hosts to move between networks with maintained connectivity. This article describes multihomed Mobile IP enabling mobile hosts to register multiple care-of addresses at the home agent, to enhance the performance of wireless network connectivity. A prototype is described and a simulator evaluation shows the performance of our approach.
\end{abstract}

\section{Introduction}

In future wireless local area networks (WLAN), connectivity to access points (AP) by different technologies and different providers will be a reality. Technologies like 802.11 [1], 802.16 [2] and HiperLAN [3] will support wireless network connectivity to wired network infrastructures, to reach the Internet and for other types of services. WLAN-technologies are becoming efficient enough to support network capabilities for applications running in desktop computers.

With the use of WLANs, new challenges arise and mobile hosts $(\mathrm{MH})$ will face multiple APs with possibly different capabilities and utilization.

The work described in this article is based on the $802.11 \mathrm{~b}$ technology. In infrastructure mode the association with an AP is based on link-layer mechanisms using the signal quality. The selection is invisible to upper layer protocols and one association at a time is possible.

The selection of which AP to associate with should also be available for higher level protocols, the applications and the users. It might be that the signal quality is somewhat better to one AP but the overall performance is better at another. Then it is reasonable to use the AP with the best overall performance.

In the largest study so far [4], a university campus equipped with WLANs is evaluated. 476 APs are spread over 161 buildings divided into 81 subnets. 5,500 students and 1,215 professors are equipped with laptops. The study shows that $17 \%$ of the sessions involved handover and that $40 \%$ of it is between different subnets,

P. Dini et al. (Eds.): SAPIR 2004, LNCS 3126, pp. 197-206, 2004.

(C) Springer-Verlag Berlin Heidelberg 2004 
causing the IP traffic to fail. MHs sometimes perform frequent handovers between APs while being in the same place.

To manage handover between networks without disrupting flows, the Mobile IP (MIP) [5] is proposed and partly deployed. For an MH connected to the home network, the IP will operate normally. If the $\mathrm{MH}$ disconnects from the home network and connects to a foreign network, the MIP will manage network mobility which will be transparent for the protocol layers above the network layer and to the user of the MH. There are two versions of MIP: MIPv4 [6] and MIPv6 [7].

The study [4] shows the MIP requirements and the potential to associate with multiple APs simultaneously to avoid breaking and disrupting sessions. Wireless connections are prone to errors and by using multiple simultaneous connections to APs, a more reliable connectivity can be achieved.

The work in this article describes an approach to enhanced network connectivity to MHs connecting to WLANs by evaluating network layer characteristics. The MIP is extended to support the multihomed connectivity. A prototype developed is also described. This will enable the AP selection on other criteria than just the signal-tonoise ratio (SNR). Traffic to and from an $\mathrm{MH}$ can be sent using multiple APs.

Section 2 describes multihomed MIP and its prototype. Section 3 describes the simulator evaluation. In section 4 related work is presented and section 5 concludes the paper and discusses future work.

\section{Multihomed Mobile IP}

This section shortly described a prototype and discusses the changes made to MIPv4, where a modified registration mechanism is used. The route optimization is also altered to be sent from the $\mathrm{MH}$ to correspondent hosts $(\mathrm{CH})$ (as with MIPv6). For a closer description see [16].

Multihomed MIP enhances the performance and reliability for MHs connecting to WLANs. Wireless connections are prone to errors and changing conditions which must be considered to enable applications for desktop computers to be usable on MHs connecting wireless.

The multihoming is managed by the MIP and hidden from the IP routing, keeping IP routing unaware. For a sender, multihomed MIP can be considered an any-cast approach [8] where a sender relies on the network protocol to use the best available destination for the packets. The available destination will be one of possibly multiple care-of addresses used by an MH. In IPv6, an any-cast address is used to reach the best available destination (server) among multiple destinations supporting the service required. The approach in this paper for a sender to any-cast address an $\mathrm{MH}$, is that the MH's home address is used to locate the best care-of address. The difference from the any-cast approach in IPv6 is that it is address-based instead of server-based and the destination will be the same host.

The MH keeps a list of all networks with valid advertisements and registers the care-of address at the HA (and the $\mathrm{CH}$ if route optimization is used) for the networks supporting the best connectivity. To evaluate the connectivity, the $\mathrm{MH}$ monitors the deviation in arrival times between agent advertisements and calculates the metric based on this information (see formula 1). This metric is used to describe the MH's 
connectivity to foreign networks. A small metric indicates that agent advertisements sent at discrete time intervals arrive without collisions and without being delayed by

$$
\begin{gathered}
\text { SampleDelta }=\text { CurrentArrivalTime }- \text { LastArrivalTime. } \\
\text { MeanDelta }=\text { SampleDelta } \times \delta+\text { MeanDelta } \times(1-\delta) . \\
\text { Metric }=(\text { SampleDelta }- \text { MeanDelta })^{2} \times \mu+\text { Metric } \times(1-\mu) .
\end{gathered}
$$

the FA. This indicates available bandwidth as well as the FA's capability to relay traffic for the $\mathrm{MH}$. Among the care-of addresses registered at the HA, the FA with the smallest metrics will be installed as the default gateway in the $\mathrm{MH}$.

The selection of which care-of address to use for an $\mathrm{MH}$ is based on the delay between a $\mathrm{CH}$ or the $\mathrm{HA}$ and the $\mathrm{MH}$, where the delay includes wireless links. In IP routing with protocols like RIP and OSPF a wireless last hop link is not considered in the route calculation. A hop count of 1 is used in the RIP protocol, and a static link cost in OSPF based on the link (usually Ethernet) connecting the APs. In multihomed MIP, IP routing is used to the care-of address selected but the selection of what careof address to use is managed by MIP. The HA makes its own selection and the $\mathrm{CH}$ does the same if route optimization is used.

The measurements and metric calculations of the deviation are made prior to registration and maintained while being registered at the foreign network. An MH is configured with the maximum number of care-of-addresses to register. Since the $\mathrm{MH}$ may register multiple associations with foreign networks, the $\mathrm{HA}$ and $\mathrm{CHs}$ can have multiple bindings for an MH's home address. Based on the round trip time (RTT) between the $\mathrm{HA} / \mathrm{CH}$ and the $\mathrm{MH}$, one of the care-of addresses will be installed as the tunnel end-point to the MH. The measuring of RTTs is based on the messages sent between the $\mathrm{MH}$ and the $\mathrm{HA}$ or the $\mathrm{CH}$.

The choice of care-of address is based on individual selections by the $\mathrm{HA}$, the $\mathrm{CH}$ and the $\mathrm{MH}$ for packets sent by them. In a scenario where an $\mathrm{MH}$ has registered three care-of addresses and there are two $\mathrm{CHs}$, one using the HA to communicate with the $\mathrm{MH}$ and the other using route optimization, three different APs may be used: one by the $\mathrm{HA}$, another by the $\mathrm{CH}$ using route optimization and the third by the $\mathrm{MH}$ to send packets (see figure 1).

The metrics for the selection of care-of address made by the HA and $\mathrm{CH}$ (if route optimization is managed by the $\mathrm{MH}$ ) is based on the Jacobson/Karels algorithm [16] (se algorithm 2). A small value is preferred.

$$
\text { Difference }=\text { SampleRTT }- \text { EstimatedRTT } \text {. }
$$

$$
\begin{gathered}
\text { EstimatedRTT }=\text { EstimatedRTT }+(\delta \times \text { Difference }) . \\
\text { Deviation }=\text { Deviation }+\delta(\mid \text { Difference } \mid- \text { Deviation }) . \\
\text { Metric }=\mu x \text { EstimatedRTT }+\phi x \text { Deviation. }
\end{gathered}
$$




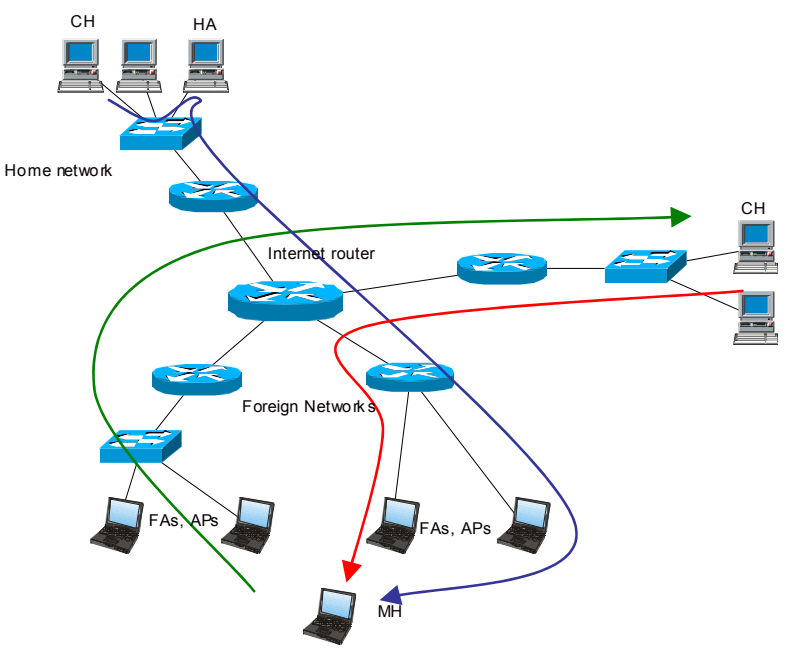

Fig. 1. A multihomed connectivity scenario where the $\mathrm{HA}, \mathrm{CH}$ and $\mathrm{MH}$ make their own selections of which care-of address to use.

To avoid rapid changes resulting in flapping of the care-of addresses and the default gateway because of metrics close in value, a new care-of address or gateway is only chosen if its value is less than the value used minus a threshold.

The registration request and binding update messages are modified by adding an $\mathrm{N}$-flag to the flag field, to enable the HA and the $\mathrm{CH}$ to distinguish between a nonmultihomed and a multihomed registration/update. An $\mathrm{HA}$ and a $\mathrm{CH}$ receiving the messages with an $\mathrm{N}$-flag will keep the existing bindings for the $\mathrm{MH}$. The $\mathrm{MH}$ monitors the time between registration requests and registration replies and calculates the RTT. The RTT is added as an extension in the next registration request. The HA will maintain all registrations for an $\mathrm{MH}$ and based on the metrics it will install a tunnel into the forwarding table with the care-of address with the smallest metrics. With binding updates the $\mathrm{CH}$ will itself measure the RTT. The processing in the $\mathrm{MH}$ is shown in figure 2 and the processing in the HA is shown in figure 3.

\section{Performance Evaluations}

The evaluation uses the GlomoSim[10] network simulator. The node MH2 equipped with two wireless interfaces associates on different channels to a network where it can reach FAs in different subnetworks. A Constant Bit Rate (CBR) flow of 1 Mbps is generated between $\mathrm{MH} 2$ and the $\mathrm{CH}$ in both directions. Other $\mathrm{CBR}$ flows are periodically generated between FA1 and $\mathrm{MH} 1$ as well as between FA2 and MH2 to add extra load on the FAs and the medium (see figure 4).

The evaluation in figure 5 shows that the selection of FAs based on the SNR could lead to less throughput, than if the selection is based on network-level measurements. The SNR value $(22 \mathrm{~dB})$ is the same for all curves although the traffic through FAs increases. The throughput from the $\mathrm{CH}$ to $\mathrm{MH} 2$ for different additional loads from 
$\operatorname{var} \mathrm{N}_{\text {foreign }}$ : set of available fa and announced care-of address;

$\mathrm{N}_{\text {reg }}$ : set of registered care-of addresses;

$\mathrm{M}_{\mathrm{adv}}$ : array of calculated metrics;

$\mathrm{T}_{\text {regReq }}$ : array of clock times for RTT measurements;

$A_{\text {adv }}$ : set of agent advertisements received;

Processing a < agent advertisement, fa, care-of-address $>$ message: begin

receive $<$ agent advertisement, fa, care-of-address $>$;

if fa $\notin \mathrm{N}_{\text {foreign }}$ then begin

$\mathrm{N}_{\text {foreign }}:=\mathrm{N}_{\text {foreign }} \cup\{\mathrm{fa}$, care-of-address $\}$;

$\mathrm{M}_{\mathrm{adv}}[\mathrm{fa}]:=$ initilize;

if $\left|N_{\text {reg }}\right|<\max$ care-of addresses to register then begin

$\mathrm{N}_{\text {reg }}:=\mathrm{N}_{\text {reg }} \cup\{$ fa, care-of-address $\}$;

if $\left|\mathrm{N}_{\mathrm{reg}}\right|>1$ then

set(n-flag)

else

clear(n-flag);

send $<$ registration request, home-address, ha, care-of-address, $n$-flag, $0>$ to ha via fa; $\mathrm{T}_{\text {regReq }}[\mathrm{fa}]:=$ clock

end

else if <agent advertisement, fa, care-of-address $>\notin \mathrm{A}_{\mathrm{adv}}$ then

$\mathrm{M}_{\mathrm{adv}}[\mathrm{fa}]:=$ calculated metric according to formula 1 ;

end

$A_{a d v}:=A_{a d v} \cup<$ agent advertisement, fa, care-of-address $>$

Processing a $<$ registration reply, home-address, ha $>$ message: begin

receive $<$ registration reply, home-address, ha $>$ from fa;

end

receive $<$ registration reply, ho
$\mathrm{T}_{\text {regReq }}[\mathrm{fa}]:=$ clock $-\mathrm{T}_{\text {regReq }}[\mathrm{fa}]$

Time expires for a binding to a fa: begin

if $\left|\mathrm{N}_{\text {reg }}\right|>1$ then

set(n-flag)

else

clear(n-flag);

send $<$ registration request, home-address, ha, care-of-address, $n$-flag, $\mathrm{T}_{\text {regReq }}[\mathrm{fa}]>$ to ha via fa; end

$\mathrm{T}_{\text {regReq }}[\mathrm{fa}]:=$ clock

Time expires, compare $\mathrm{N}_{\text {reg }}$ and $\mathrm{N}_{\text {foreign }}$ : begin

if $\min \left\{\mathrm{M}_{\mathrm{adv}}[\mathrm{w}]: \mathrm{w} \in \mathrm{N}_{\text {foreign }} \wedge \mathrm{w} \notin \mathrm{N}_{\mathrm{reg}}\right\}<\max \left\{\mathrm{M}_{\mathrm{adv}}[\mathrm{w}]: \mathrm{w} \in \mathrm{N}_{\mathrm{reg}}\right\}$ - threshold then begin

fa $:=\left\{\mathrm{w}: \min \left\{\mathrm{M}_{\mathrm{adv}}[\mathrm{w}]\right\} \wedge \mathrm{w} \in \mathrm{N}_{\text {foreign }} \wedge \mathrm{w} \notin \mathrm{N}_{\text {reg }}\right\}$;

$\left\{\mathrm{fa}_{\min }\right.$, care-of-address $\left.\mathrm{min}_{\min }\right\}:=\left\{\{\mathrm{x}, \mathrm{y}\}:\{\mathrm{x}, \mathrm{y}\} \in \mathrm{N}_{\text {foreign }} \wedge \mathrm{x}=\mathrm{fa}\right\}$

$\mathrm{fa}:=\left\{\mathrm{w}: \max \left\{\mathrm{M}_{\mathrm{adv}}[\mathrm{w}]\right\} \wedge \mathrm{w} \in \mathrm{N}_{\mathrm{reg}}\right\}$

$\left\{\mathrm{fa}_{\max }\right.$, care-of-address $\left.\mathrm{max}_{\max }\right\}:=\left\{\{\mathrm{x}, \mathrm{y}\}:\{\mathrm{x}, \mathrm{y}\} \in \mathrm{N}_{\mathrm{reg}} \wedge \mathrm{x}=\mathrm{fa}\right\}$;

$\mathrm{N}_{\text {reg }}:=\mathrm{N}_{\text {reg }} \backslash\left\{\mathrm{fa}_{\max }\right.$, care-of-address $\left.\mathrm{max}_{\max }\right\} ; \mathrm{N}_{\text {reg }}:=\mathrm{N}_{\text {reg }} \cup\left\{\mathrm{fa}_{\min , \text { care-of-address }}\right.$ min $\}$;

if $\left|N_{\text {reg }}\right|>1$ then

set(n-flag)

else

clear(n-flag);

send $<$ registration request, home-address, ha, care-of-address ${ }_{\min ,} \mathrm{n}$-flag, $0>$ to ha via $\mathrm{fa}_{\min }$;

end

$\mathrm{T}_{\text {regReq }}\left[\mathrm{fa}_{\mathrm{min}}\right]:=$ clock

end

Fig. 2. The processing of MIP messages in the MH 


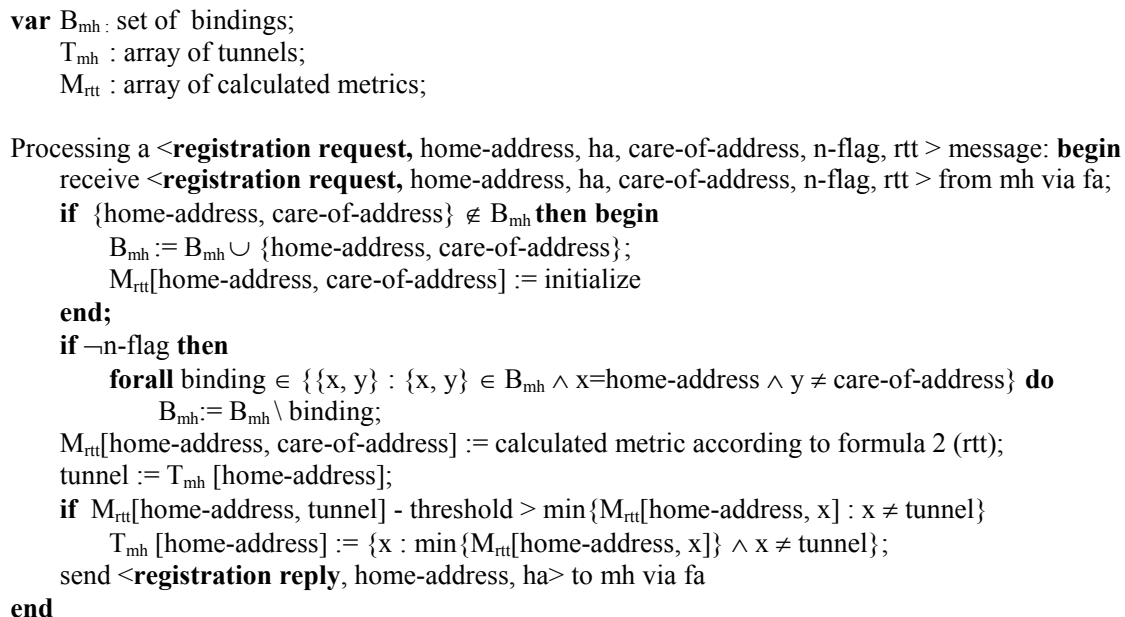

Fig 3. The processing of registration requests in the HA.

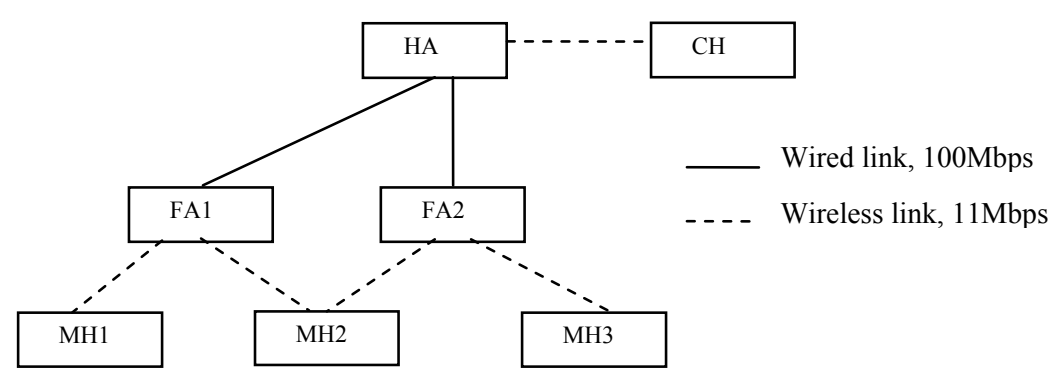

Fig 4. The topology used for evaluation. An MH reaches two FAs in different subnetworks and communicates with a $\mathrm{CH}$ in its home network. $\mathrm{MH} 1$ and $\mathrm{MH} 2$ are used to add additional flows to the FAs.

MH1 and MH3 is shown. The bandwidth of the additional loads is used to name the curves in the graph.

We evaluated two approaches for MIP messages; one where MIP messages had higher priority than the flows, and the other where they had the same priority. Figure 6 shows that the best result is achieved with MIP messages using the same priority as the flows.

The "2FA (c)" curve shows the throughput when MIP messages has higher (control) priority than ordinary traffic. The "2FA (r)" curve shows the throughput with the same priority (real time) for all traffic. The low metrics in bandwidth shown at times 15, 30 and 45 seconds are explained by the time it takes to react on bad metrics when a recently good connection becomes congested. 


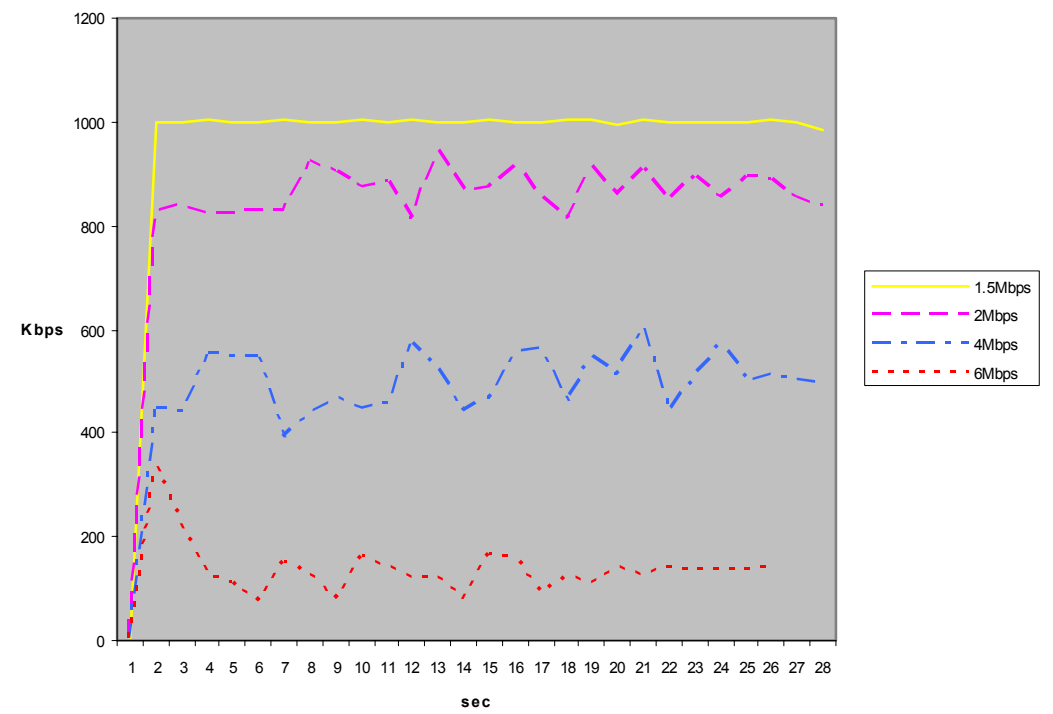

Fig. 5. Throughput from $\mathrm{CH}$ to the $\mathrm{MH}$ with additional loads between $\mathrm{MH}-\mathrm{Load}$ and the FA.

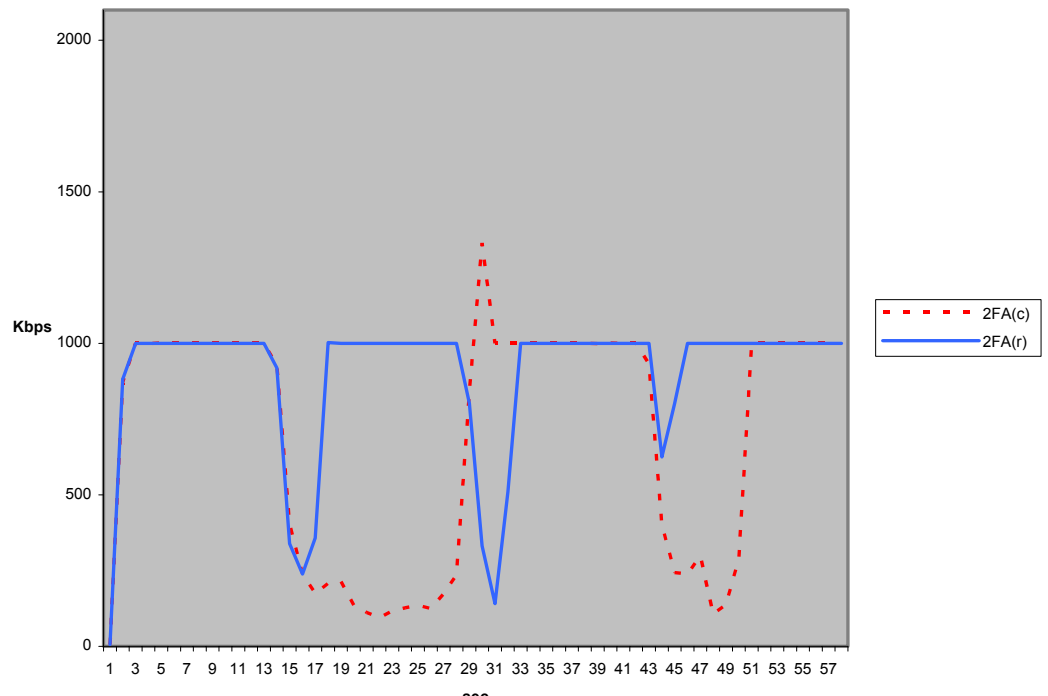

Fig. 6. Throughput from the $\mathrm{CH}$ to $\mathrm{MH} 2$ with multihoming based on different priorities of MIP messages. 
Figure 7 shows the throughput from the $\mathrm{CH}$ to $\mathrm{MH} 2$ with different FA selection strategies. The same traffic pattern is used as in the simulation shown in figure 6 . The "SNR" curve shows the throughput when an FA is selected based on the SNR. The curve named "1 FA" shows the throughput when one FA is selected as both the gateway and the care-of address for the $\mathrm{MH}$ based on dynamic metrics.

With one FA the traffic to and from the MH will be forwarded through the same FA. With multihoming one FA may forward traffic to the MH and the other from the $\mathrm{MH}$. The throughput achieved with multihoming is shown by the curve named "2 FA".

With multihomed MIP, the MH with multiple FAs can easily switch between FAs to enhance throughput even further. The evaluation shows the best results with multihomed MIP.

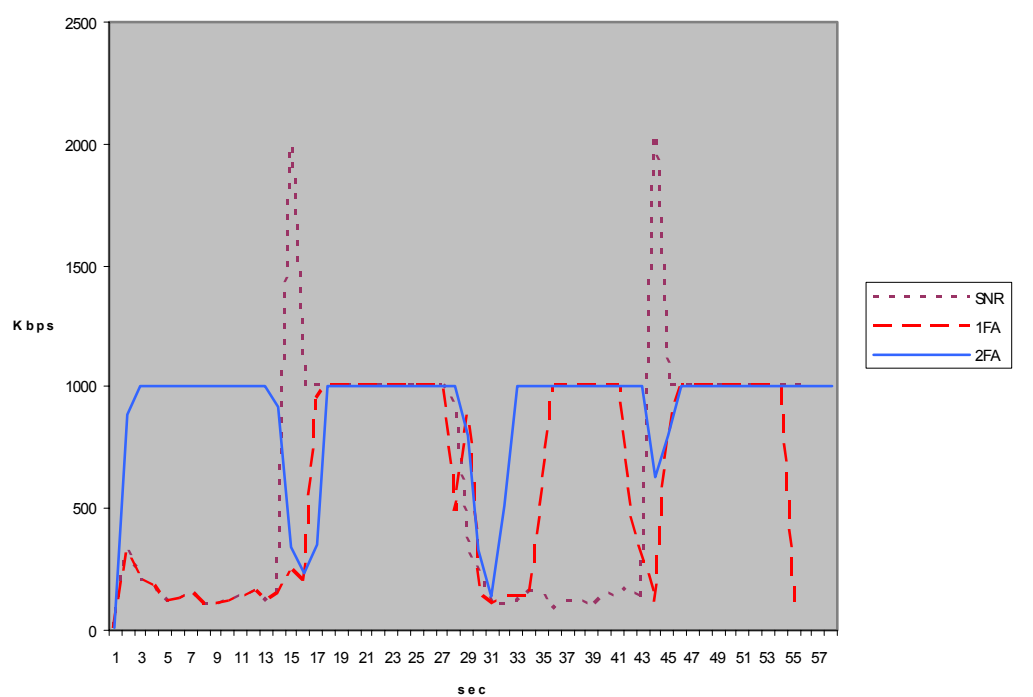

Fig. 7. Throughput between the $\mathrm{CH}$ and $\mathrm{MH} 2$ with different FA selection algorithms

\section{Related Work}

In MIPv4, an option for simultaneous bindings is proposed for sending packets to multiple care-of addresses for an $\mathrm{MH}$. Packets will be duplicated at the HA and one copy sent to each registered care-of address, so that packets can be received through multiple APs. This option was proposed to decrease the number of dropouts of packets during handover, and for an $\mathrm{MH}$ with bad connections to APs to receive the same packet through several APs, with an increased probability of a success. The solution does not enable the network layer to decide which connection to use and it will waste resources in the WLAN. 
In the current specification of MIPv6, all traffic uses the same care-of address. This prevents the dynamics of the MIP from fully utilizing the dynamics in WLANs and should be altered.

In [11], an approach to multihoming for survivability is proposed, managed at the datalink layer and based on radio signalling. This approach restricts the selection of APs to the datalink layer and is not available to higher levels.

In [12], a transport layer protocol is proposed striping data between multiple links to achieve bandwidth aggregation. The work presented in this paper instead aims to evaluate multiple connections and how to use the best available connection(s) to forward packets.

Another transport layer solution is presented in [13] for multihomed hosts. Here the sender selects one of the host's IP addresses as the destination address for the packets. If the IP address becomes unavailable due to network failure, the protocol will switch to another IP address for the same destination host with maintained connectivity at the transport layer. The approach does not address delays considering a wireless last hop link.

In [14] the correlation of signal-to-noise-ratio and throughput is shown. However the approach does not cover multiple MHs communicating using e.g 802.11 (using clear to send, request to send and NAV times to avoid collisions). In this case the SNR will not be sufficient, since SNR is not affected. Traffic measurements are instead required.

In [15] a proposal for multihoming with MIPv6 is proposed. Our proposal is intended for both MIP versions. We also propose a care-of address selection algorithm and evaluate its performance. In [15] no selection algorithm is presented.

\section{Conclusion and Future Work}

The proposed approach describes extended MIP to manage multiple simultaneous connections with foreign networks. Based on the registered care-of-addresses, multiple paths can be used for packets to and from an MH. This approach will also prevent MHs from flapping between foreign networks due to the fact that an $\mathrm{MH}$ has similar quality of connectivity to multiple APs. The proposed approach achieves enhanced throughput, more reliable and efficient connectivity. The current prototype is based on MIPv4 but can easily be applied to MIPv6 as well; future work will describe this.

The association should be made available to higher protocol layers and provided through an Application Programmer's Interface (API). Future work will look into possible solutions to achieve this.

The multihomed approach in MIP is also being tested and evaluated for connectivity between wired IP networks and ad hoc networks. The journal paper [17] describes the architecture proposed for this. 


\section{References}

1. Gast, M.S.: 802.11 Wireless Networks, The Definitive Guide. O'Reilly (2002)

2. Eklund, C., Marks, R.B, Stanwood, K.L: IEEE Standard: A Technical Overview of the WirelessMAN Air Interface for Broadband Wireless Access. IEEE Communications Magazine, No 6 (2002) 98-107

3. van Nee, R.D.J., Awater, G.A., Morikura, M., Takanashi, H., Webster, M.A., Halford, K.W.: New High-Rate Wireless LAN Standards. IEEE Communications Magazine, Volume 37, No 12 (1999) 82-88

4. Kotz, D., Essien, K.: Analysis of a Campus-wide Wireless Network. Mobicom (2002) 107 118

5. Perkins, C.: Mobile IP. IEEE Communications Magazine (May 2002) 66-82

6. Perkins, C.: IP Mobility Support for IPv4, revised. IETF RFC3220 (2002)

7. Johnson, D.B., Perkins, C.E.: Mobility Support in IPv6. draft-ietf-mobileip-ipv6-18.txt (2002)

8. Metz, C.: IP Anycast Point-to(Any) Point Communication. Internet Computing, Volume 6, No 2 (2002) 94-98

9. Peterson, L.L., Davie, B.S: Computer Networks a Systems Approach. Morgan Kaufman Publishert (2000) 391-392

10.GlomoSim http://pcl.cs.ucla.edu/projects/glomosim/

11.Dahlberg, T.A, Jung, J.: Survivable Load Sharing Protocols: a Simulation Study. Wireless Networks, Volume 7, Issue 3. (2001) 283-296

12.Hsieh, H.-Y., Sivakumar, R.: A Transport Layer Approach for Achieving Aggregate Bandwidths on Multi-homed Mobile Hosts. Mobicom (2002) 83-94

13.Stewart, R., Metz, C.: SCTP: New Transport Protocol for TCP/IP. Internet Computing Volume 5, No 6 (2001) 64-69

14.Hiroto, A., Tamura, Y., Tobe, Y., Tokuda, H.: Wireless Packet Scheduling with Signal-toNoise Ratio Monitoring. IEEE Conference on Local Computer Networks, (2000) 32-41

15.Nagami, K., et al.: Multi-homing for small scale fixed network Using Mobile IP and NEMO. draft-nagami-mip6-nemo-multihome-fixed-network-00.txt (2004)

16.Ahlund, C., Zaslavsky, A.: Multihoming in Mobile IP. IEEE International Conference on High Speed Networks and Multimedia Communications, (2003) Lecture Notes in Computer Science (LNCS), Springer-Verlag.

17.Ahlund, C., Zaslavsky, A.: Extending Global IP Connectivity for Ad Hoc Networks. Telecommunication Systems, Modeling, Analysis, Design and Management, Volume 24, Nos. 2-4, Kluwer publisher 